\title{
Anti-bias Passive Localization for Fixed Target Using Bearings-only
}

\author{
Shun Sun ${ }^{1, a}$, Chen Guo ${ }^{1, b}$ \\ ${ }^{1}$ Institute of Information Fusion, Naval Aeronautical and Astronautical University, Yantai 264001, \\ China. \\ asunms7@qq.com, bguoguo8908743@163.com
}

Keywords: bearings-only, passive localization, system error.

\begin{abstract}
An anti-bias passive localization algorithm is proposed to solve the problem of single motion platform passive localization based on bearing-only for fixed emitter target when system bias exists. Bearing difference measurements are constructed through analysis of bearings-only measurements. After linear localization equations which are established according to the geometric relationship between the target and the observer station is analysed, anti-bias localization algorithm based on the constraint total least square is proposed through derivation of error terms. Simulation results indicate the proposed algorithm can locate target effectively under system error, and it can be extend to situation of multi-stations.
\end{abstract}

\section{Introduction}

Passive sensor have advantages of good concealment, long detection distance, applicability, etc., but due to dimension limitation of measurement information of a single sensor (only measuring angles or only distance), it is difficult to estimate target state effectively, which get widely attention from scholars at home and abroad [2, 3]. Bearings-only localization with single observer station for a fixed source target is a common passive localization scenario, not only the random error of bearing measurement, but also the system error, such as calibration bias, equipment bias, should be considered. System error is typically modeled as constant during observation period. Ignoring the impact of the system error may lead to wrong state estimation, even generating false target. Therefore, it is necessary to study the bearings-only localization in system error condition.

Currently registration algorithms at measurement level mainly contain real-time online registration algorithm [4] and offline batch processing registration algorithm. The latter can be divided into maximum likelihood registration (MLR) algorithm [5,6] and least square registration (LSR) algorithm [7]. However, the system error is regarded as parameter to be estimated in the above algorithms, and is estimated jointly with the target state, which increases deminsion of estimation vector, slows down convergence speed of algorithm and reduces accuracy after convergence. Meanwhile part of the algorithms are only applicable to small system error situation, large system error may lead to less effective registration and even divergence. Single station bearings-only anti-bias location algorithm is obtained by bearing measurment differential and constrained total least squares (CWLS) algorithm, which prevent the above problems effectively.

\section{Scenario Description}

In 2D localization scenario, we assume that state vector of the unknown fixed source target is $\boldsymbol{u}=\left[\begin{array}{ll}x_{t} & y_{t}\end{array}\right]^{\mathrm{T}}$ and that of observer station is $\boldsymbol{s}(k)=\left[\begin{array}{ll}x_{s}(k) & y_{s}(k)\end{array}\right]^{\mathrm{T}}$ where $k=1,2, \cdots, N$. Then, bearing measurement at time $k$ is

$$
\bar{\beta}(k)=\arctan \left(\frac{y_{t}-y_{s}(k)}{x_{t}-x_{s}(k)}\right)
$$

In fact, the actual measurements data include random noise and sensor bias, so actual bearing measurement model is situation

$$
\beta(k)=\bar{\beta}(k)+\Delta \beta+n(k)
$$


where $\Delta \beta$ denotes system error which is regarded as constant during the observation period, $n(k)$ denotes random noise error which is Gaussian distribution with zeros mean and its variance is $\sigma_{\beta}^{2}$.

Since system error can be regared as constant, difference operation can be used to eliminate constant bias. Bearing difference can be constructed as

$$
\theta(k)=\beta(k)-\beta(1)=\bar{\beta}(k)-\bar{\beta}(1)+n(k)-n(1)=\bar{\theta}(k)+n_{e}(k)
$$

where $\bar{\theta}(k)=\bar{\beta}(k)-\bar{\beta}(1)$ is true bearing difference measurements relative to $\bar{\beta}(1), n_{e}(k)=n(k)-n(1)$ is equivalent random noise error.

Defining all measurement set before time $k$ is $\mathbf{Z}(k)=[\theta(1), \theta(2), \cdots, \theta(k)]$, then $\mathbf{Z}(k)=\overline{\mathbf{Z}}(k)+\boldsymbol{v}(k)$ where definition of $\overline{\mathbf{Z}}(k)$ is similar to $\mathbf{Z}(k), v(k)=\left[\begin{array}{llll}n_{e}(1) & n_{e}(2) & \cdots & n_{e}(k)\end{array}\right]^{\mathrm{T}}$ is error vector whose covariance matrix is

$$
\boldsymbol{R}_{\theta}=E\left[\boldsymbol{v}(k) \boldsymbol{v}(k)^{\mathrm{T}}\right]=\left[\begin{array}{cccc}
2 \sigma_{\beta}^{2} & \sigma_{\beta}^{2} & \cdots & \sigma_{\beta}^{2} \\
\sigma_{\beta}^{2} & 2 \sigma_{\beta}^{2} & \cdots & \sigma_{\beta}^{2} \\
\vdots & \vdots & \ddots & \vdots \\
\sigma_{\beta}^{2} & \sigma_{\beta}^{2} & \cdots & 2 \sigma_{\beta}^{2}
\end{array}\right]
$$

\section{Localization based on CTLS}

Bearings-only localization has translated into bearing difference localization through method of the above section. K. Doğancay studied robot localization based on landmark bearings, which is similar to the localization scenario this paper proposed. Typical localization scenario is shown in Fig. 1.

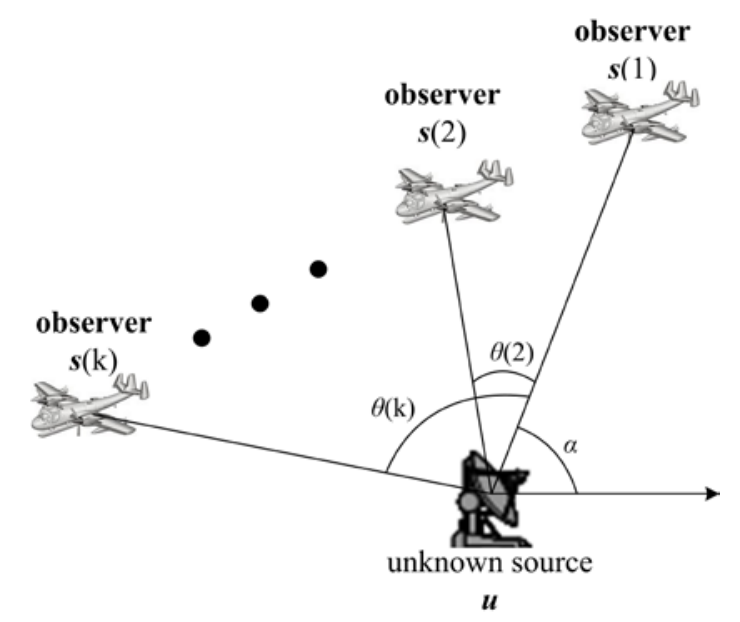

Fig. 1 Typical localization scenario schematic

According to the geometric position relationship between the observer station and the source target, we have

$$
\tan (\bar{\theta}(k)+\alpha)=\frac{y_{s}(k)-y_{t}}{x_{s}(k)-x_{t}}
$$

where $\alpha$ denotes an unknown orientation angle parameter.

Then, linear localization equation can be obtained as

$$
\left[\begin{array}{c}
x_{s}(k) \sin \bar{\theta}(k)-y_{s}(k) \cos \bar{\theta}(k) \\
x_{s}(k) \cos \bar{\theta}(k)+y_{s}(k) \sin \bar{\theta}(k) \\
\sin \bar{\theta}(k) \\
-\cos \bar{\theta}(k)
\end{array}\right]^{\mathrm{T}}\left[\begin{array}{c}
-\boldsymbol{R}(\alpha) \boldsymbol{u} \\
\cos \alpha \\
\sin \alpha
\end{array}\right]=0
$$

or compact form

$$
\overline{\boldsymbol{a}}(k) \boldsymbol{X}(k)=0
$$

where rotation matrix $\boldsymbol{R}(\alpha)$ is 


$$
\boldsymbol{R}(\alpha)=\left[\begin{array}{cc}
\cos \alpha & \sin \alpha \\
-\sin \alpha & \cos \alpha
\end{array}\right]
$$

For all measurements, the above equation can be extended as

$$
\overline{\boldsymbol{A}}(k) \boldsymbol{X}(k)=0
$$

where $\overline{\boldsymbol{A}}(k)=\left[\overline{\boldsymbol{a}}(k)^{\mathrm{T}}, \overline{\boldsymbol{a}}(k)^{\mathrm{T}}, \cdots, \overline{\boldsymbol{a}}(k)^{\mathrm{T}}\right]^{\mathrm{T}}$.

Since actual measurements $\theta(k)$ have random noise, K. Doğancay and others use the total least squares (TlS) to solve the above equations and obtain the unknown source position estimate $\hat{\boldsymbol{u}}$. But TLS method ignores the correlation between the column vectors of matrix $\bar{A}(k)$, so the solution should be established as the constrain total least squares (CTLS). Substituting Eq. 3 into $\bar{A}(k)$, we have

$$
\begin{aligned}
& \boldsymbol{A}(k)=\overline{\boldsymbol{A}}(k)+\Delta \boldsymbol{A}(k) \\
& \Delta \boldsymbol{A}(k)=\left[\begin{array}{llll}
\boldsymbol{E}_{1}(k) v(k) & \boldsymbol{E}_{2}(k) v(k) & \boldsymbol{E}_{3}(k) v(k) & \boldsymbol{E}_{4}(k) v(k)
\end{array}\right] \\
& \boldsymbol{E}_{1}(k)=\operatorname{diag}\left(x_{s}(1) \cos \bar{\theta}(1)+y_{s}(1) \sin \bar{\theta}(1), x_{s}(2) \cos \bar{\theta}(2)+y_{s}(2) \sin \bar{\theta}(2), \cdots, x_{s}(k) \cos \bar{\theta}(k)+y_{s}(k) \sin \bar{\theta}(k)\right)(12) \\
& \boldsymbol{E}_{2}(k)=\operatorname{diag}\left(y_{s}(1) \cos \bar{\theta}(1)-x_{s}(1) \sin \bar{\theta}(1), y_{s}(2) \cos \bar{\theta}(2)-x_{s}(2) \sin \bar{\theta}(2), \cdots, y_{s}(k) \cos \bar{\theta}(k)-x_{s}(k) \sin \bar{\theta}(k)\right) \\
& \boldsymbol{E}_{3}(k)=\operatorname{diag}(\cos \bar{\theta}(1), \cos \bar{\theta}(2), \cdots, \cos \bar{\theta}(k)) \\
& \boldsymbol{E}_{4}(k)=\operatorname{diag}(\sin \bar{\theta}(1), \sin \bar{\theta}(2), \cdots, \sin \bar{\theta}(k))
\end{aligned}
$$

where $\operatorname{diag}(\cdot)$ indicates diagonal matrix.

Then Eq. 9 can be rewriten as

$$
\begin{aligned}
& \boldsymbol{A}(k) \boldsymbol{X}(k)=\Delta \boldsymbol{A}(k) \boldsymbol{X}(k)=\boldsymbol{C}(k) v(k) \\
& \boldsymbol{C}(k)=\left[\boldsymbol{E}_{1}(k) \boldsymbol{X}_{1}(k)+\boldsymbol{E}_{2}(k) \boldsymbol{X}_{2}(k)+\boldsymbol{E}_{3}(k) \boldsymbol{X}_{3}(k)+\boldsymbol{E}_{4}(k) \boldsymbol{X}_{4}(k)\right]
\end{aligned}
$$

where $\boldsymbol{X}_{\boldsymbol{i}}(k)$ denotes the $i$ th element of vector $\boldsymbol{X}(k)$.

The weighting matrix can be defined as

$$
\boldsymbol{W}(k)=E\left(\boldsymbol{C}(k) \boldsymbol{v}(k) \boldsymbol{v}(k)^{\mathrm{T}} \boldsymbol{C}(k)^{\mathrm{T}}\right)=\boldsymbol{C}(k) \boldsymbol{R}_{\theta} \boldsymbol{C}(k)^{\mathrm{T}}
$$

And CTLS can be established as

$$
\begin{cases}\min _{\boldsymbol{X}} & \boldsymbol{X}(k)^{\mathrm{T}} \boldsymbol{A}(k)^{\mathrm{T}} \boldsymbol{W}(k)^{-1} \boldsymbol{A}(k) \boldsymbol{X}(k) \\ \text { s.t. } & \boldsymbol{X}(k)^{\mathrm{T}} \boldsymbol{\Omega}(k) \boldsymbol{X}(k)=\text { const }\end{cases}
$$

where $\boldsymbol{\Omega}(k)=E\left(\Delta \boldsymbol{A}(k)^{\mathrm{T}} \boldsymbol{W}(k)^{-1} \Delta \boldsymbol{A}(k)\right)$, const denotes and non-negative constant.

Substituting $\Delta \boldsymbol{A}(k)$ into $\boldsymbol{\Omega}(k)$, we have

$$
\boldsymbol{\Omega}(k)=\left[\left\{\operatorname{tr}\left(\boldsymbol{E}_{m}^{\mathrm{T}}(k) \boldsymbol{W}(k)^{-1} \boldsymbol{E}_{n}(k) \boldsymbol{R}(k)\right)\right\}_{m n}\right]
$$

where $\{\cdot\}_{m n}$ denotes $4 \times 4(\mathrm{mn})$ submatrix for $m, n=1,2,3,4$.

Lagrange multiplier method [9] can be used to solve the above constraint minimization problem. The minimum generalized eigenvalue $\lambda_{\min }$ corresponding generalized eigenvalue vector $\boldsymbol{d}_{\text {min }}$ can be obtained through generalized eigenvalue decomposition of $\left(\boldsymbol{A}(k)^{\mathrm{T}} W(k)^{-1} \boldsymbol{A}(k), \boldsymbol{\Omega}(k)\right)$. The parameters $\hat{\boldsymbol{X}}(k)$ can be estimated as

$$
\boldsymbol{X}(k)=\frac{\boldsymbol{d}_{\min }}{\left|\boldsymbol{d}_{\min }(3: 4)\right|}
$$

with $|\cdot|$ denoting the Euclidean norm. Estimations of orientation angle parameter and target position are

$$
\begin{aligned}
& \hat{\alpha}(k)=\arctan \left(\hat{\boldsymbol{X}}_{4}(k) / \hat{\boldsymbol{X}}_{3}(k)\right) \\
& \hat{\boldsymbol{u}}(k)=-\boldsymbol{R}(\hat{\alpha}(k))^{\mathrm{T}} \hat{\boldsymbol{X}}_{1: 2}(k)
\end{aligned}
$$


Parameter vector $\boldsymbol{X}(k)$ is required to be known when the weighted matrix $\boldsymbol{W}(k)$ is calculated. We can use $\hat{\boldsymbol{X}}(k-1)$ instead of $\boldsymbol{X}(k)$ if $\hat{\boldsymbol{X}}(k-1)$ is obtained, otherwise make $\boldsymbol{W}(k)$ as identity matrix. In addition, actual measurements can be used instead of true measurements to calculate error terms $\Delta \boldsymbol{A}(k)$.

\section{Simulation and analysis}

We assume a motion platform in the two-dimensional simulation scenario utilizes bearings-only to locate a fixed target localization. The fixed source target locate at $\boldsymbol{u}=[0 \mathrm{~km}, 0 \mathrm{~km}]^{\mathrm{T}}$. The motion platform is initially at $s(1)=[2 \mathrm{~km}, 10 \mathrm{~km}]^{\mathrm{T}}$ and is moving at a constant speed of $300 \mathrm{~m} / \mathrm{s}$ at an angle of $225^{\circ}$ with respect to the $\mathrm{x}$ axis. Bias and root mean square error (RMSE) of target position estimation as below.

$$
\begin{aligned}
& \operatorname{Bias}(k)=\frac{1}{M} \sum_{i=1}^{M}\left(\boldsymbol{u}-\hat{\boldsymbol{u}}^{i}(k)\right) \\
& \operatorname{RMSE}(k)=\sqrt{\frac{1}{M} \sum_{i=1}^{M}\left|\boldsymbol{u}-\hat{\boldsymbol{u}}^{i}(k)\right|^{2}}
\end{aligned}
$$

where, $M$ is the Monte Carlo times, $\boldsymbol{u}$ is the true target position, $\hat{\boldsymbol{u}}^{i}(k)$ is the estimation target position in $i$ th Monte Carlo experiment at time $k$.

Fig. 2 gives the performance of proposed algorithm as syetem error is $2^{\circ}$ and measurement error standar deviation is $0.1^{\circ}$ and $0.5^{\circ}$, respectively. Fig. 2 (a) shows that, as time progresses, localization bias of the proposed algorithm drops rapidly to zero, then tends to stable. When the measurement error is small, it need less time to reach the stable state quickly. Fig. 2 (b) shows that RMSE of the proposed algorithm is convergent and the smaller the measurement error, the faster the convergence. The observation time of target in simulation is $60 \mathrm{~s}$ but average running time is $0.2 \mathrm{~s}$, so the algorithm is good real-time. The above analysis shows that the proposed algorithm can locate fixed source target effectively under the existence of system error, and the positioning result is asymptotic unbiased. To further verify the performance of the algorithm, the algorithm performance at time $k=60$ is investigated under the conditions of different measurement errors and system errors.

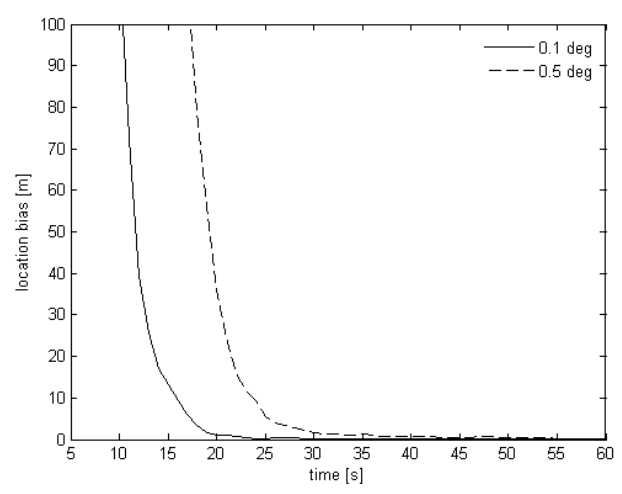

(a) Location bias

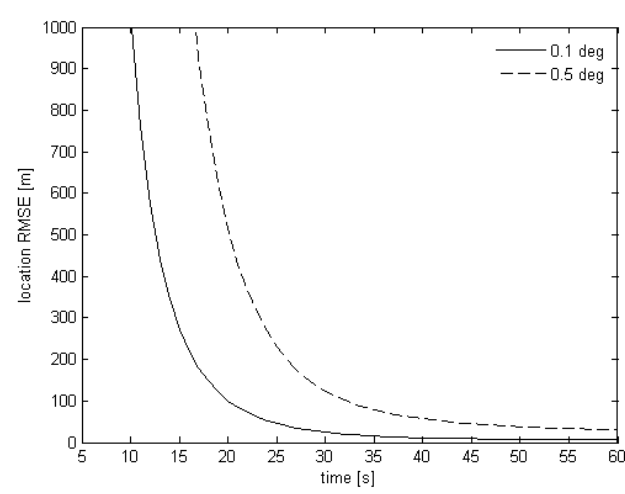

(b) Location RMSE

Fig.2 Performance of the proposed algorithm

Table 1 gives the performance of proposed algorithm at time $\mathrm{k}=60$ as syetem error is $2^{\circ}$ and measurement error standar deviation is $0.1^{\circ}, 0.5^{\circ}, 1.0^{\circ}, 2.0^{\circ}$ and $3.0^{\circ}$ respectively. Table 2 gives the proposed algorithm performance when measurement error standar deviation is $0.5^{\circ}$ and syetem error is $1^{\circ}, 2^{\circ}, 3^{\circ}, 4^{\circ}$ and $5^{\circ}$ respectively. As Table 1 and Table 2 show that, bias and RMSE of the proposed algorithm increase as measurement error increases, but change little as system error increases. 
Table 1 Performance of the proposed algorithm as measurement error changes

\begin{tabular}{cccccc}
\hline Mesurement error [ ${ }^{\circ}$ ] & 0.1 & 0.5 & 1.0 & 2.0 & 3.0 \\
\hline Bias [m] & 0.1307 & 0.3129 & 0.6323 & 1.4057 & 1.8365 \\
RMSE [m] & 6.1984 & 30.6577 & 61.7231 & 123.1245 & 186.5780 \\
\hline
\end{tabular}

Table 2 Performance of the proposed algorithm as system error changes

\begin{tabular}{cccccc}
\hline system bias [ ${ }^{\circ}$ ] & 1 & 2 & 3 & 4 & 5 \\
\hline Bias [m] & 0.0653 & 0.2139 & 0.1447 & 0.2755 & 0.1946 \\
RMSE [m] & 30.9223 & 30.9602 & 30.8413 & 30.6106 & 30.8633 \\
\hline
\end{tabular}

\section{Summary}

In order to solve the problem of single motion platform passive localization based on bearing-only for fixed emitter target, bearing difference measurements are constructed, and error terms of linear localization equations are derived, then anti-bias localization method based on CTLS is proposed. Simulation results indicate the proposed method can locate target effectively under system error and performance of the method is insusceptible to bias. It is valuable to extend the method to applications. The proposed method can be extended to the case of multiple motion platforms by simple deduction.

\section{References}

[1]. Y. He, J. J. Xiu, J. W. Zhang, et al. Radar Data Processing With Applications (Second Edition). Beijing: Publishing House of Electronics Industry, 2009, p. 209-210.

[2]. J. C. Chen, L. Yip, J. Elson, et al. Coherent acoustic array processing and localization on wireless sensor networks. Proceeding of the IEEE. Vol. 91 (2003) No. 8, p. 1154-1162.

[3]. J. Q. Fang, D. Z. Feng, J. Li. A robustly convergent algorithm for source localization using time difference of arrival and frequency difference of arrival. Journal of Electronics \& Information Technology. Vol. 37 (2015) No. 4, p. 798-803.

[4]. Z. T. Hu, Y. M Hu, J. Zhang, et al. Joint estimation of system error and state based on cubature Kalman filter. Journal of Optoelectronics • Laser. Vol. 26 (2015) No. 3, p. 535-540.

[5]. O. Nickens, R. Branko. Maximum likelihood registration for multiple dissimilar sensors. IEEE Transactions on Aerospace and Electronic Systems. Vol. 39 (2003) No. 3, p. 1074-1083.

[6]. W. H. Wu, J. Jiang. Maximum likelihood registration for passive sensors of multiple airborne platforms in WGS-84. Systems Engineering and Electronics. Vol. 37 (2015) No. 2, p. 304-309.

[7]. C. W. Qu, C. H. Wang, Z. Xu. NLS-based registration algorithm for bearings-only location by multiple airborne observers. Signal Processing. Vol. 28 (2012) No. 4, p. 533-538.

[8]. K. Doğancay. Exploiting geometric translations in TLS based robot localization from landmark bearings. 17th European Signal Processing Conference. Glasgow, 2009, p. 95-99.

[9]. K. C. Ho, Y. T. Chan. An asymptotically unbiased estimator for bearings-only and Doppler-bearing target motion analysis. IEEE Transactions on Signal Processing. Vol. 54 (2006) No. 3, p. 809-822. 